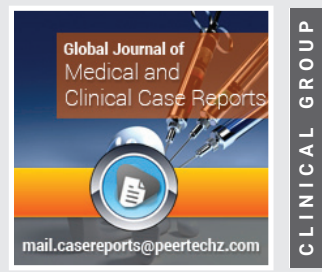

\section{Aesthetic and functional smile} enhancement using digital

\section{occlusion equilibration \& dental} GPS smile designing concepts -

\section{A clinical report}

\author{
Sagar Abichandani ${ }^{1 *}$, Neha Abichandani² and Divya \\ Venkatachalam ${ }^{3}$ \\ ${ }^{1}$ Managing Director, Silverline Dent and Care Solutions Pvt. Ltd., Proprietor, Mumbai, Maharashtra, \\ India \\ Executive Director, Silverline Dent and Care Solutions Pvt. Ltd., Chief Dentist, Mumbai, Maharashtra, \\ India
}

${ }^{3}$ Associate Dentist at The Dental Hub, Mumbai, Maharashtra, India
Received: 30 March, 2020

Accepted: 23 June, 2020

Published: 25 June, 2020

*Corresponding author: Sagar Abichandani, Managing Director, Silverline Dent and Care Solutions Pvt. Ltd., Proprietor, Mumbai, Maharashtra, India,

E-mail: sagar.abichandani@gmail.com

https://www.peertechz.com

(A) Check for updates

\title{
Abstract
}

An aesthetic and functional smile enhancement has great contribution to a person's self confidence in addition to their longevity and functional success. It gives us an idea of treatment modality as a corrective approach when trying to uplift the patient's confidence and giving her a reason to smile again.

\section{Introduction}

The application of principles of aesthetics to the natural or artificial teeth and restoration is dental aesthetics (GPT 1999). Aesthetic dentistry is characterized primarily by the smile. The goal in aesthetic dentistry is to achieve or create a natural dentition with a beautiful smile. Smile designing can be achieved either by restorative, orthodontic, periodontal approaches or a combination approach.

A proper diagnosis of the case gives a proper guideline for treatment planning and achieving the results of patient's desire.

\section{Case presentation}

The following is a case report of a patient treated using Dental GPS. Concept with minimal invasion. A 26-year old patient, consulted with a chief complaint of unpleasant veneers which were bulky and poor in appearance, with respect to upper front teeth; having past dental history of undergoing ceramic veneers at another clinic wherein, she was dissatisfied with the approach as well as the final restorative result, and wanted us to address the concern. Her dental hygiene habits included, brushing once daily, without usage of dental floss and mouthwash [1].

\section{On Extra - oral examination (Figure 1)}

No pain, clicking, crepitation, or deviation of the mandible was noted. The facial profile, dental and facial midlines were parallel and were perpendicular to the inter-pupillary line. The lips were competent with a prominent chin. But, due to maxillary teeth an increased upper lip support was noted.

\section{Intra-oral examination (Figure 2)}

On Intraoral examination, generalized gingival 
inflammation was seen alongwith stains and calculus present with soft edematous gingiva. Disappropriately fabricated veneers from 13 to 23 with no shade matching and missing smile curve. Dental caries with 17. Deep developmental pits with 12, 22 [2].

\section{Periodontal Assessment:}

Prognosis:

The prognosis is favourable after scaling and root planing with restoration of optimal health.

Prognosis is favourable after the replacement of old veneers and restoration of smile.

Treatment:

\section{Options presented To patient}

- Scaling and root planing for oral prophylaxis.

- Replacement with new ceramic veneers for upper front teeth.

- Composite fillings with 17 and palatal pits in the cingulum area relation to 12,22.

Treatment Plan:

- Oral prophylaxis with scaling and root planing.

- Evaluation of occlusal prematuries ( if any) with T-Scan and elimination of the same before any definitive work begins.

- Replacement of existing veneers with ceramic veneers \&
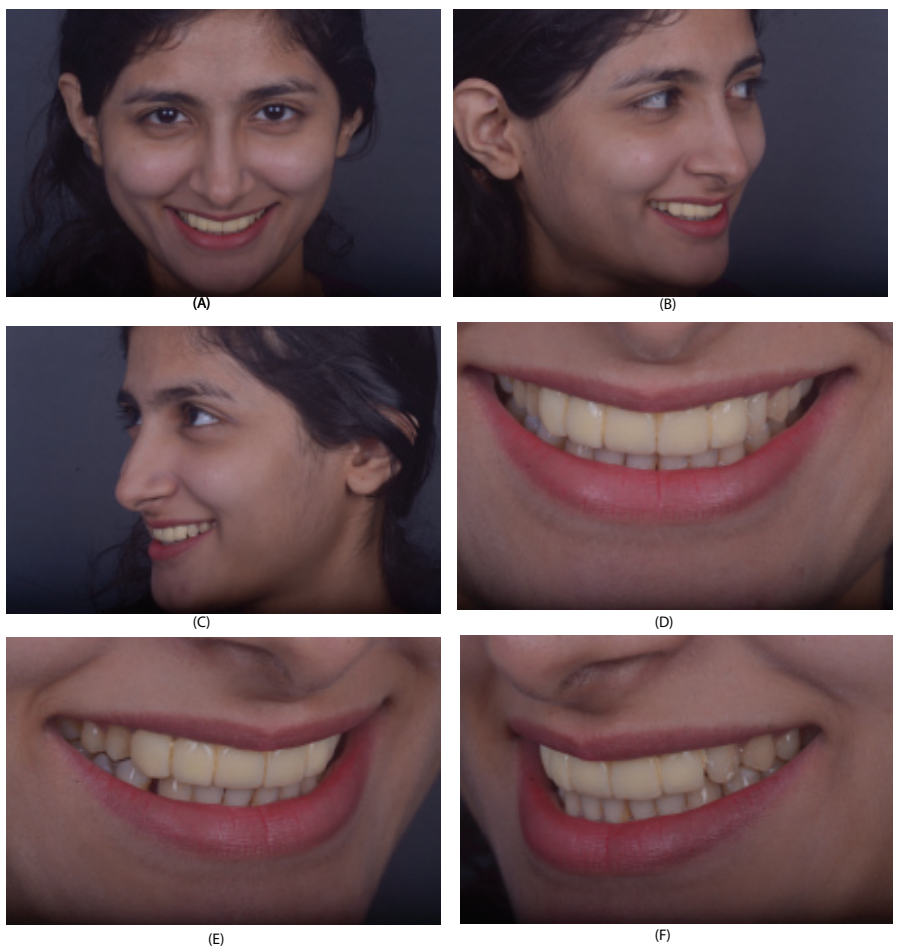

Figure 1: Pre- Op Extra Oral Pictures.

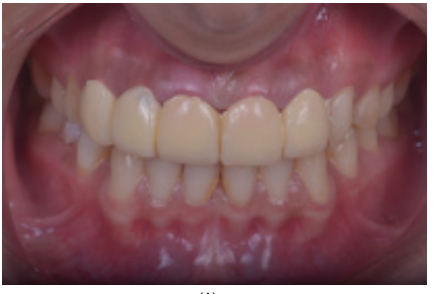

(A)
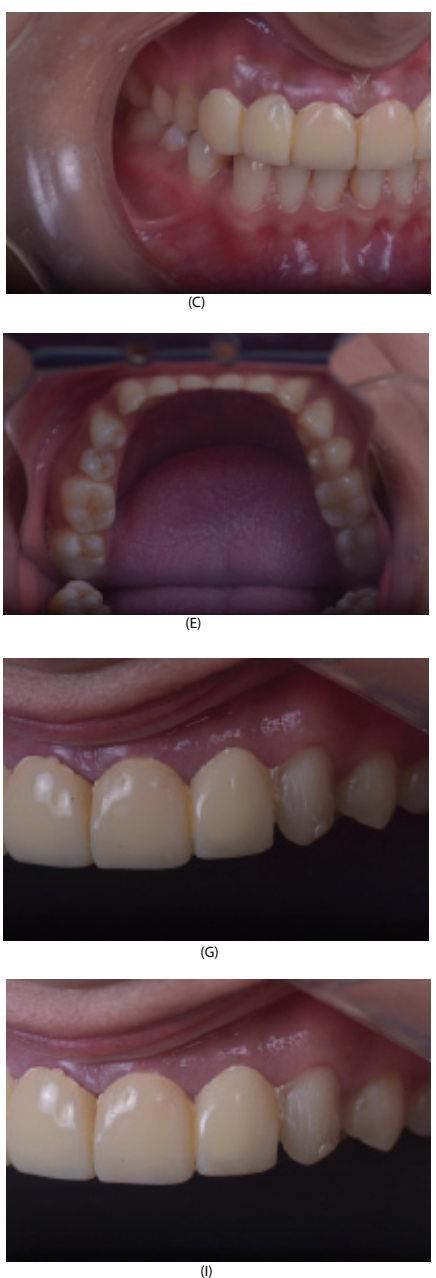

Figure 2: Pre- Op Intra Oral Pictures.

including both premolars too in the planning phase for maintenance of symmetry, proportions and balance in the smile.

- Gingivectomy to balance the gingival zeniths on the left and right.

- Composite filling on the occlusal surface of 17 and pits in the cingulum in relation to 12,22 .

\section{Treatment sequence}

Patient's Bite analysis was carried out with T-Scan to remove any prematurities which was found to be in relation to tooth 16 (Figure 3a). Periodontal probing was carried out to identify the probing depth and if any gum corrections would need osseous recontouring as well (to maintain the biological width). 
A complete set of photographs were taken alongwith diagnostic casts mounted on a semiadjustable articulator using a facebow record. Two sets diagnostic impression was recorded with a combination of putty consistency elastomeric impression (Affinis, COLTENE) and light body consistency elastomeric impression (Affinis, COLTENE) was taken with double mix - single stage technique.

The patient's occlusal pattern was noted before the waxup for the upper front 10 teeth [3].

Oral prophylaxis was carried out in the form of scaling \& root planing. Composite fillings was done on the occlusal surface of 17 and pits in the cingulum area in relation to 22,12 .

Since the patient was highly apprehensive as to how she would look, we decided to use Dental GPS concept for getting the patient acceptance \& commencement of the treatment( Figure 3). Since the dimension ratio of present veneers were not appropriate and the patients smile line extends till the premolars, patient accepted, consented for ceramic veneers from 15 to 25 alongwith gingivectomy in relation to $11,13,15,21,23,25$.

Gingivectomy was performed with soft tissue diode laser (Biolase,CA,USA) in relation to 11,13,15,21,23,25 (Figure 4).

Simultaneously, a Digital Facebow concept (Methot A. Facial Proportions. CJCD 2006) was used to print the M lines (Figure 5) and doing a guided wax up with the help of tooth coloured inlay casting wax (Dental restoration material inlay wax, ZOGEAR, China). Silicone index (putty consistency elastomeric impression material) was recorded of the finished wax up for the fabrication of the temporaries and for guiding in the tooth reduction.

The previous veneers were carefully sectioned \& removed to prevent any inadvertent loss of tooth structure. On examination, it was seen that tooth preparation was aggressive and patient had sensitivity in few areas of her teeth. The sharp areas of the tooth preparation were finished and polished (no further tooth preparation was carried out) (Figure 6).

An immediate chairside mockup was carried out from the new laboratory waxup according to the APT (Aesthetic Pre Evaluative

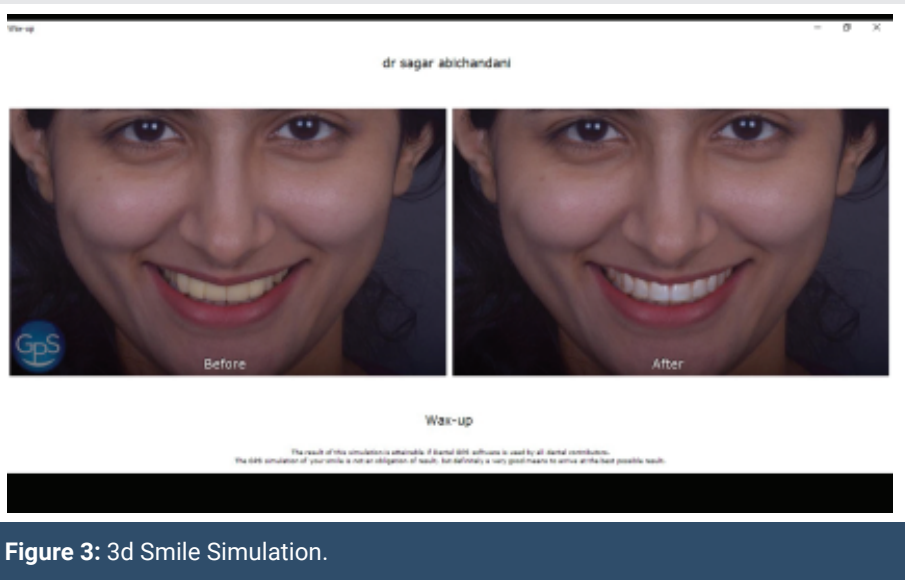

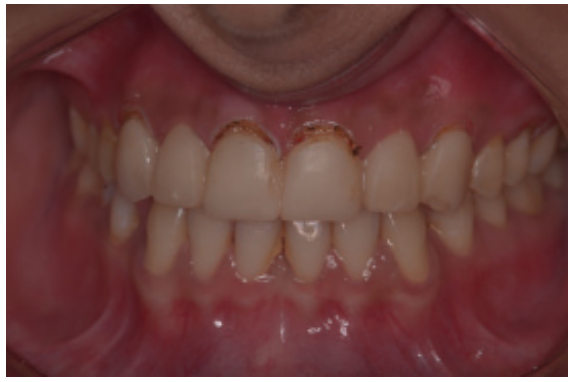

Figure 4: Digital Occlusal Equilibration.
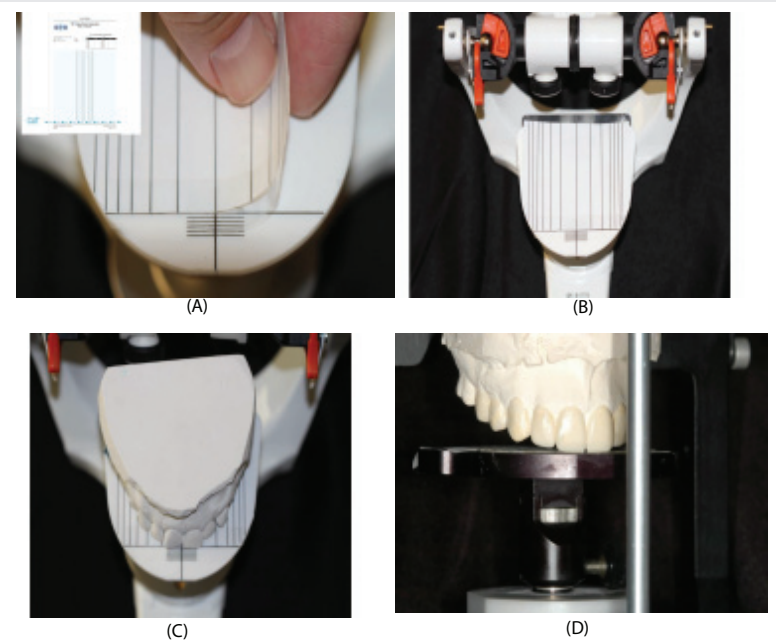

(D)

Figure 5: Digital Facebow \& Mounting on Semi Adjustable Articulator

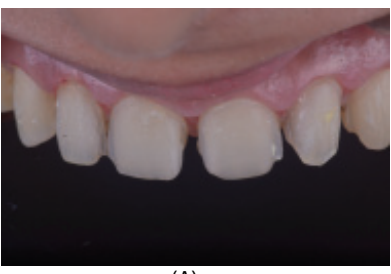

(A)
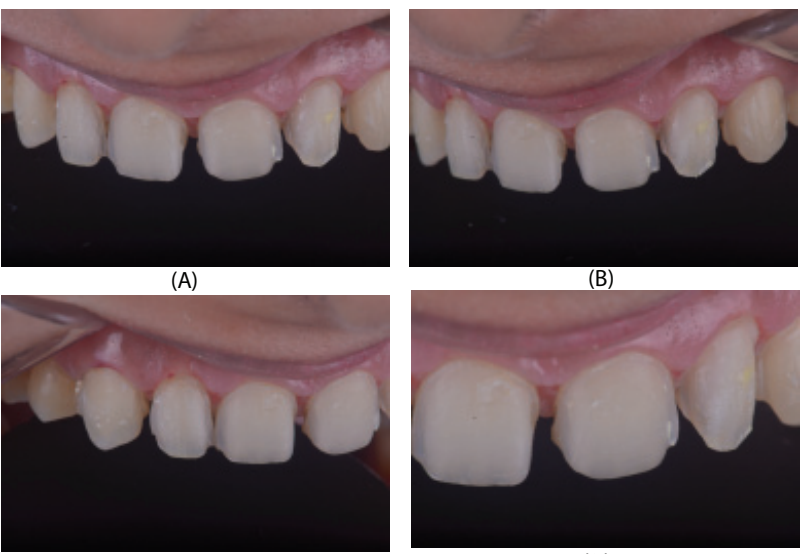

(C)

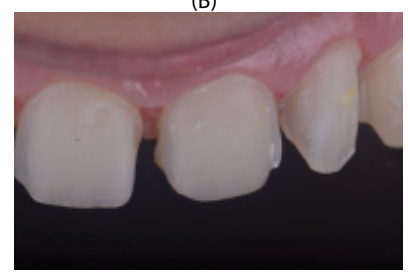

(D)

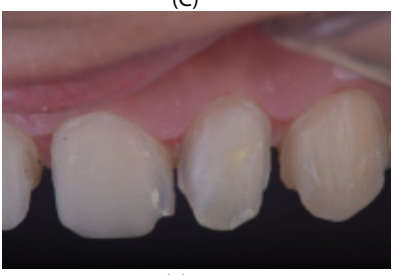

(E)

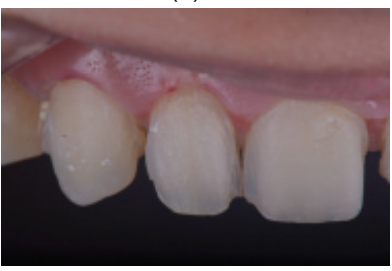

$(\mathrm{F})$

Figure 6:Laser Gingivectomy, Removal of Old Veneers.

Temporaries) technique (Galip Gurel's Concept for evaluation of function, esthetics and phonetics) (Figure 7).

The patient loved the new look and was now confident that her new smile will be better than the existing one [4]. 
On subsequent recall, the look and function was acceptable without any discomfort or complaint. The tooth preparation was carried out through the temporaries for minimally invasive dentistry protocols to preserve the enamel and be as conservative as possible (after placement of retraction cords) in tooth reduction (Figure 8 ). $2^{\text {nd }}$ set of retraction cords (Ultrapak, Ultradent) impregnated with hemostatic agent (Racestyptine solution, Septodont) were then placed into the gingival sulcus and elastomeric impressions were taken. Irreversible hydrocolloid material was used for a check cast impression and provisionals (Cooltemp, COLTENE) were fabricated, checked for its fit, occlusal interference, esthetics, phonetics, trimmed, polished and cemented (Temposil, COLTENE). Final Impressions were taken with Elastomeric impression materials ( Affinis, COLTENE). Shade selection was done (VITA toothguide 3D- Master, VITA) and temporaries refabricated and spot bonded.

Monolithic lithium disilicate ceramic veneers (EMax, Ivoclar Vivadent,Switzerland) with an incisal cutback were fabricated.

Ceramic layering was done for the incisal one-third of the veneers with a combination of enamel shades, translucent
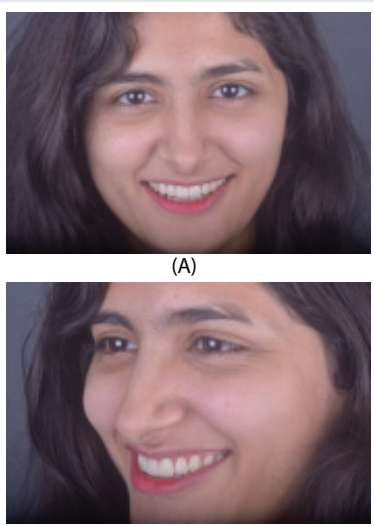

(C)
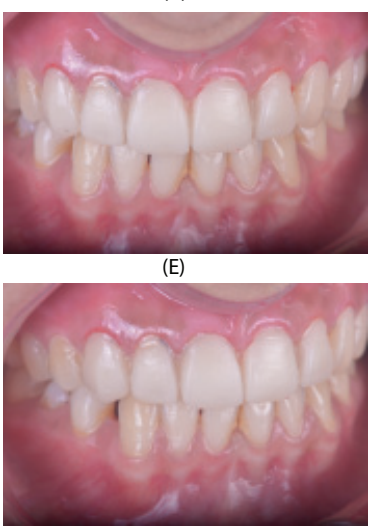

(G)

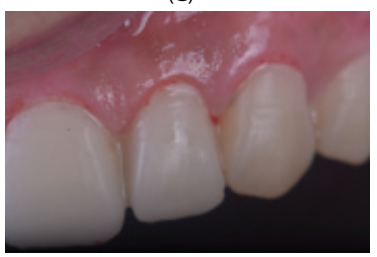

(I)
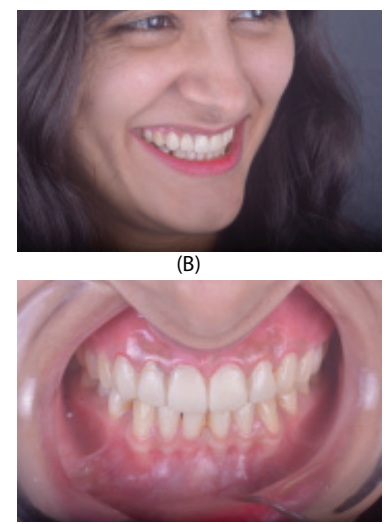

(D)

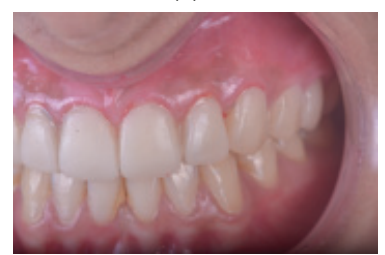

(F)

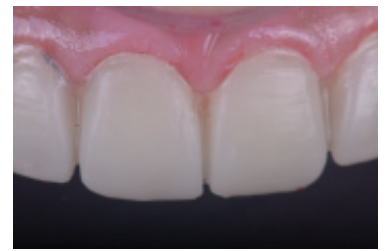

(H)

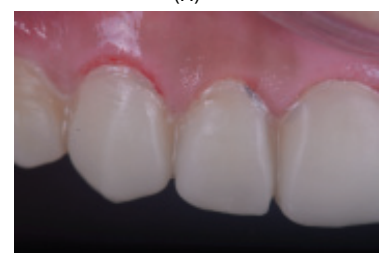

(J)

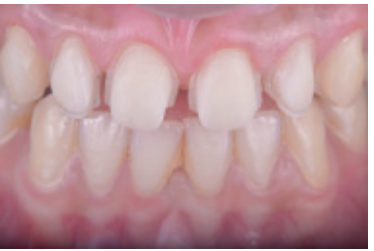

(A)

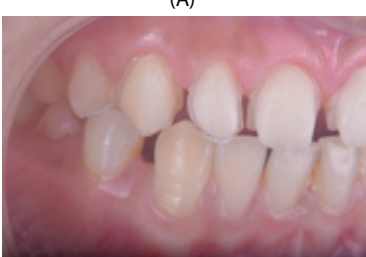

(C)
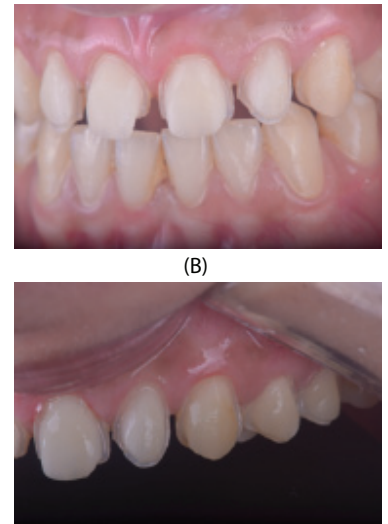

(D)
Figure 8: Guided Tooth Preparation Using Apt Technique.

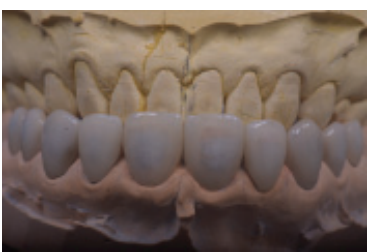

(A)

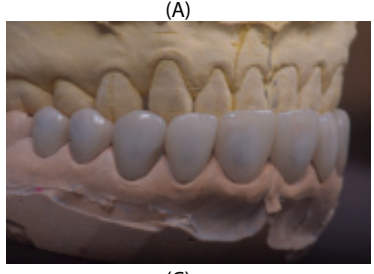

(C)

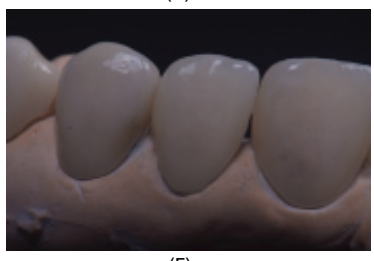

(E)

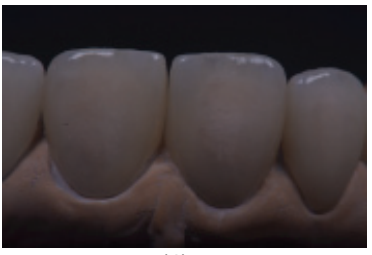

(G)

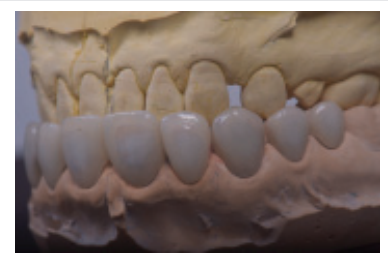

(B)

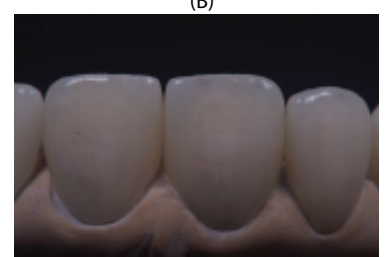

(D)

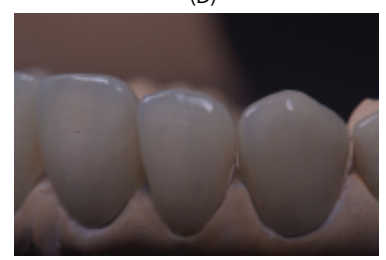

(F)

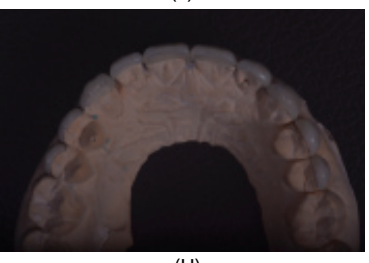

(H)
Figure 9: Layered Lithium Disilicate Restorations Fabricated.

and stains in line with the clinical pictures and lab notes for characterization (Figure 9). Occlusion was verified again with articulating paper. Ceramic. Veneers were cemented using Variolink Veneer Cement ( Ivoclar Vivadent) under rubber dam isolation. Excess cement was removed and dental floss was passed interproximally (Figure 10). Oral hygiene instructions were given. Patient was recalled after 3 month and reevaluated for maintenance of the oral hygiene instructions (Figure 11).

\section{Discussion}

Since the underlying tooth colour wasn't planned to 
be changed, we opted for minimal thickness of monolithic lithium disilicate ceramic veneers with an incisal cutback for incorporation of mamelons and incisal translucency. Dental GPS is not only a simulation software but helps us use the $2 \mathrm{D}$ image for $3 \mathrm{D}$ planning and execution with precision and accuracy with the usage of printable $M$ lines. It was imperative to win the patient's confidence after the previous veneers disheartened the patient completely, so a test smile using aesthetic prevaluative temporaries played a key role.

\section{Summary and conclusion}

Dental GPS is not only a simulation software but helps us

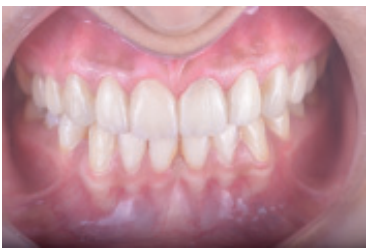

(A)

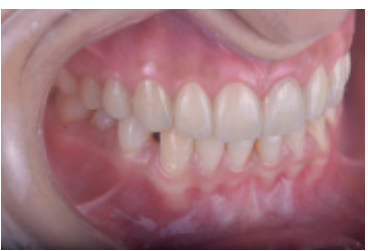

(C)

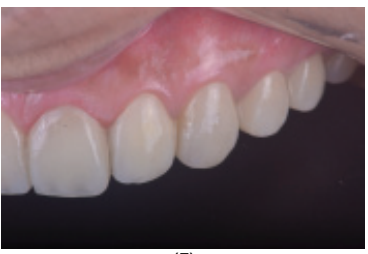

(E)

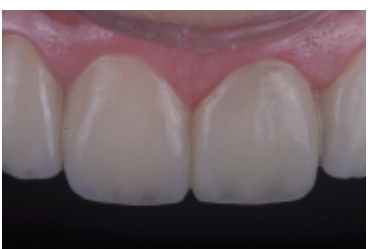

(G)

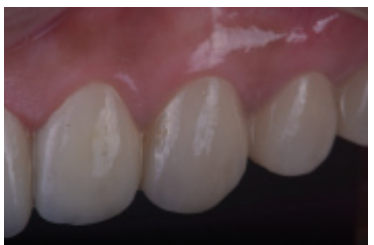

(I)

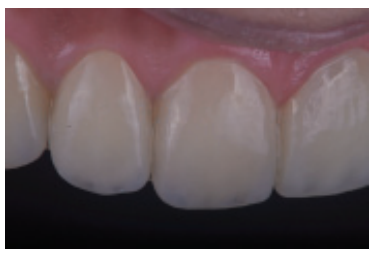

$(\mathrm{K})$

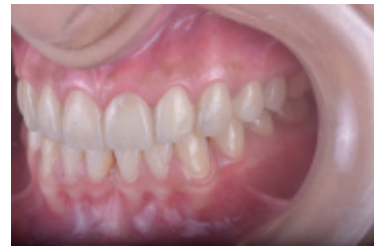

(B)

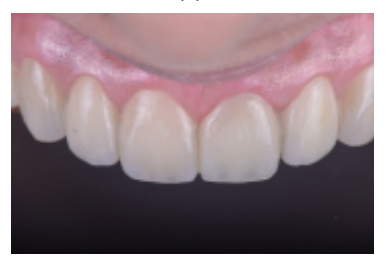

(D)

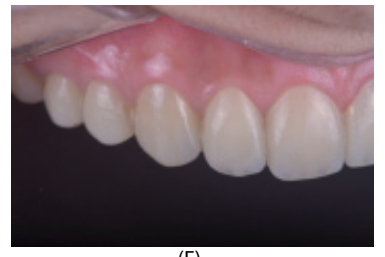

(F)

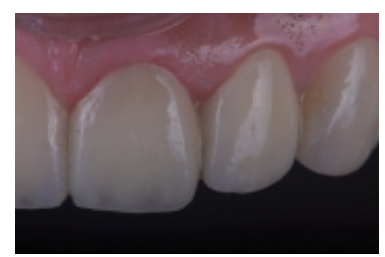

(H)

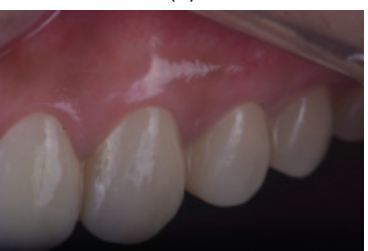

(J)

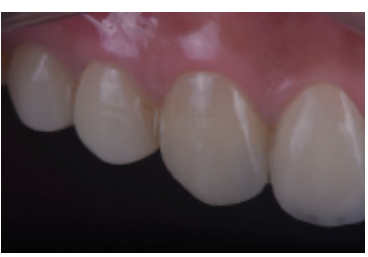

$(\mathrm{L})$

Figure 10: Final Restorations - Intra Oral Pictures.

Copyright: @ 2020 Abichandani S, et al. This is an open-access article distributed under the terms of the Creative Commons Attribution License, which permits unrestricted use, distribution, and reproduction in any medium, provided the original author and source are credited. 